\title{
3,3'-diindolylmethane downregulates cyclin D1 through triggering endoplasmic reticulum stress in colorectal cancer cells
}

\author{
XIAOBO ZHANG ${ }^{1}$, PAKIN SUKAMPORN ${ }^{2}$, SHIQIANG ZHANG $^{3}$, KYUNG-WON MIN $^{4}$ and SEUNG JOON BAEK $^{5}$ \\ ${ }^{1}$ Department of Basic Medical Science, School of Basic Medicine and Clinical Pharmacy, China Pharmaceutical University, \\ Nanjing, Jiangsu 210009, P.R. China; ${ }^{2}$ Department of Pharmacy, Faculty of Pharmacy, Mahidol University, Bangkok 10400, \\ Thailand; ${ }^{3}$ Oncology Department, Shanghai TCM-Integrated Hospital, Shanghai 200082, P.R. China; \\ ${ }^{4}$ Department of Biochemistry and Molecular Biology, Medical University of South Carolina, Charleston, SC 29425, USA; \\ ${ }^{5}$ Laboratory of Signal Transduction, College of Veterinary Medicine and Research Institute for \\ Veterinary Science, Seoul National University, Seoul 08826, Republic of Korea
}

Received September 12, 2016; Accepted May 17, 2017

DOI: $10.3892 / o r .2017 .5693$

\begin{abstract}
As a major in vivo condensation product of indole3 -carbinol, which is mostly present in cruciferous vegetables, 3,3'-diindolylmethane (DIM) has been previously reported with anti-proliferative action in different types of cancer by our group and others. To further elucidate these underlying mechanisms, we examined the effect of DIM on cyclin D1, which was aberrantly overexpressed in various cancer cells and tumors. Herein, we found that DIM downregulated cyclin D1 expression in colorectal cancer cells (CRC), which was independent of PPAR $\gamma$ expression and protease activity. Furthermore, DIM did not affect cyclin D1 mRNA expression, suggesting DIM modulated cyclin D1 expression at the translational level. Subsequently, blocking eIF2 $\alpha$ phosphorylation resulted from endoplasmic reticulum (ER) stress restored cyclin D1 in the presence of DIM. Thus, the present study demonstrates that DIM downregulates cyclin D1 through triggering ER stress in human colorectal cancer cells.
\end{abstract}

\section{Introduction}

Colorectal cancer (CRC) is one of the leading causes of cancerrelated death in the world. Phytochemicals are promising anticancer agents given their remarkable chemical structure

Correspondence to: Professor Seung Joon Baek, Laboratory of Signal Transduction, College of Veterinary Medicine and Research Institute for Veterinary Science, Seoul National University, 1 Gwanak-ro, Gwanak-gu, Seoul 08826, Republic of Korea

E-mail: baeksj@snu.ac.kr

Abbreviations: DIM, 3,3'-diindolylmethane; I3C, indole-3-carbinol; ER, endoplasmic reticulum; CRC, colorectal cancer

Key words: 3,3'-diindolylmethane, cyclin D1, ER stress, elF2 $\alpha$, PERK and diverse biological activities (1) and the prevention and treatment of cancer by dietary phytochemicals that inhibit cell growth is an exciting aspect. Subsequently, results from epidemiological studies have shown that the consumption of cruciferous vegetables could contribute to reduce the risk of CRC and other cancers, and the chemoprotective effects of cruciferous vegetables have been reported in carcinogeninduced colon cancer animal models (2).

Indole-3-carbinol (I3C) is a major bioactive component of cruciferous vegetables, such as broccoli, cabbage, brussels sprouts and cauliflower, which has been paid more attention as a cancer preventive or chemotherapeutic agent (3). As a major acid condensation product of I3C, 3,3'-Diindolylmethane (DIM) is readily detected in the liver and feces of rodents fed I3C, whereas the parent I3C was not detected in these animals, suggesting DIM may contribute to the observed physiological effects of dietary I3C. Indeed, DIM has been documented to inhibit cell proliferation and induce apoptosis in colorectal cancer cells (4), and other types of cancer cells including prostate (5), pancreas (6), breast (7), bladder (8) and hepatoma cancer (9). Mechanistically, DIM suppressed proliferation via activating peroxisome proliferator-activated receptor $\gamma$ (PPAR $\gamma$ ) and Nur77 activity in CRC cells, as well as inducing apoptosis through inactivating $\mathrm{AKT}$ and $\mathrm{NF}-\kappa \mathrm{B}$ activity in breast cancer cells (10). Moreover, DIM and its derivatives induce endoplasmic reticulum (ER) stress-mediated upregulation of death receptor 5 (DR5), causing pancreatic cancer cell apoptosis (6). We have previously reported that DIM stimulates ATF3 expression by ATF4-mediated pathway (4) which mediates apoptosis of colorectal cancer cells (2). Given both ATF3 and ATF4 are closely associated with ER stress response, DIM could trigger ER stress and subsequently induce growth inhibition and apoptosis in CRC.

Cyclin D1, a well-identified oncogenic protein, is often overexpressed in various types of cancer cells and tumors. It plays crucial roles in cell cycle machinery by activating cyclin-dependent kinase (CDK) 4/6, which subsequently phosphorylates and inactivates retinoblastoma protein $(\mathrm{pRb})$, resulting in the progression from $\mathrm{G} 1$ to $\mathrm{S}$ phase of the cell 
cycle (reviewed in ref. 11). Cyclin D1 functions as a critical regulator in DNA repair, suggesting targeting cyclin D1 may be beneficial in both pRb-negative and -positive cancer cells (12). A large number of chemicals have been shown to downregulate cyclin D1 expression in different cancer cells by triggering multiple signaling pathways (13). It has been shown that DIM downregulates cyclin D1 in breast cancer cells, which can be blocked by proteasome inhibitor (14). However, the effect of DIM on cyclin D1 expression and the underlying mechanism(s) in colorectal cancer cells remains to be investigated.

In the present study, we examined the effect of DIM on cyclin D1 expression in CRC cells, and found that DIM caused cyclin D1 downregulation independent of PPAR $\gamma$ and protease activity. Furthermore, we revealed that DIM-triggered ER stress mediated cyclin D1 translation inhibition.

\section{Materials and methods}

Reagents. DIM and cycloheximide were purchased from Sigma-Aldrich (St. Louis, MO, USA). MG132 and epoxomicin was obtained from Merck Millipore (Billerica, MA, USA). Antibodies for cyclin D1, cyclin D3, cyclin E, Ubiquitin, Actin, ATF4 were from Santa Cruz Biotechnology (Santa Cruz, CA, USA); Antibodies against CHOP, PERK and total eIF2 $\alpha$ were obtained from Cell Signaling Technology (Danvers, MA, USA). Control siRNA (\#6201) and specific siRNA against PERK (\#9024) were purchased from Cell Signaling Technology. Cell culture media were purchased from (BioWhittaker, Rockland, ME, USA). All other reagents were purchased from Thermo Fisher Scientific Inc., (Pittsburgh, PA, USA), unless others specified.

Cell culture. Human colorectal adenocarcinoma HCT-116, SW480, HT-29, LoVo and Caco-2 cells were purchased from the American Type Culture Collection (ATCC; Manassas, VA, USA). HCT-116 cells were cultured in McCoy's 5A; SW480 and LoVo were cultured in RPMI-1640 and Ham's F-12, respectively; HT-29 and Caco-2 cells were cultured in Dulbecco's modified Eagle's medium (DMEM). All media were supplemented with $10 \%$ fetal bovine serum (FBS), $100 \mathrm{U} / \mathrm{ml}$ penicillin and $100 \mu \mathrm{g} / \mathrm{ml}$ streptomycin. Cells were kept at $37^{\circ} \mathrm{C}$ under a humidified atmosphere of $5 \% \mathrm{CO}_{2}$.

Plasmid and transient transfection. Wild-type and S52A mutation of eIF2 alpha construct (pcDNA.CD2/WT-eIF2A and pcDNA.CD2/S52A-eIF2A) were kind gifts from Dr David Ron. Transient transfection was performed using PolyJet reagent (SignaGen Laboratories, Rockville, MD, USA) according to the manufacturer's instruction.

RNA interference. HCT-116 cells were seeded on 6-well plates at a density of $3.0 \times 10^{5}$ cells/well overnight. Control siRNA and siPERK (Cell Signaling Technology) was transfected at a final concentration of $100 \mathrm{nM}$ using PepMute transfection reagent (SignaGen Laboratories) according to the manufacturer's instruction.

Semi-quantitative reverse transcription PCR. Total RNA of cells was isolated by E.Z.N.A Total RNA kit (Omega Bio-Tek, Inc., Norcross, GA, USA) according to the manufacturer's protocol. Then RNA (1 $\mu \mathrm{g})$ was reverse transcribed using Verso cDNA synthesis kit (Thermo Fisher Scientific). PCR was performed using GoTaq Green Master Mix PCR Mixture (Promega, Madison, WI, USA) with primers for human cyclin D1, XBP1, ATF3 and GAPDH as follows: cyclin D1, forward, 5'-ATGGAACACCAGCTCCTGTGCTGC-3' and reverse, 5'-TCAGATGTCCACGTCCCGCACGT-3'; XBP1, forward, 5'-CCTTGTAGTTGAGAACCAGG-3' and reverse, 5'-GGGGCTTGGTATATATGTGG-3'; ATF3: forward, 5'-GTTTGAGGATTTTGCTAACCTGAC-3' and reverse, 5'-AGCTGCAATCTTATTTCTTTCTCGT-3'; GAPDH, forward, 5'-GGGCTGCTTTTAACTCTGGT-3' and reverse, 5'-TGGCAGGTTTTTCTAGACGG-3'. Thermal cycling conditions were as follows: $95^{\circ} \mathrm{C}$ for $2 \mathrm{~min}$, followed by 25 cycles of $95^{\circ} \mathrm{C}$ for $30 \mathrm{sec}, 60^{\circ} \mathrm{C}$ for $30 \mathrm{sec}$ and $72^{\circ} \mathrm{C}$ for $30 \mathrm{sec}$, and final extension at $72^{\circ} \mathrm{C}$ for $5 \mathrm{~min}$. Each PCR product was electrophoresed on agarose gel and viewed using ethidium bromide staining under ultraviolet light. The intensity of bands was analyzed by densitometry using the GAPDH band as a relative control.

Western blot analysis. Cells were washed with phosphate-buffered saline (PBS) and lysed using radio immunoprecipitation assay buffer $(50 \mathrm{mmol} / \mathrm{l}$ Tris- $\mathrm{HCl} \mathrm{pH} 7.4,150 \mathrm{mmol} / \mathrm{l} \mathrm{NaCl}$, $1 \mathrm{mmol} / 1$ EDTA, $1 \%$ Triton X-100, 1\% sodium deoxycholate, $0.1 \%$ SDS) supplemented with $1 \mathrm{X}$ protease inhibitor cocktail solution (Calbiochem, San Diego, CA, USA) and phosphatase inhibitor $\left(1 \mathrm{mmol} / 1 \mathrm{Na}_{3} \mathrm{VO}_{4}, 1 \mathrm{mmol} / 1 \mathrm{NaF}\right)$ and centrifuged at $13,000 \mathrm{x}$ for $10 \mathrm{~min}$ at $4^{\circ} \mathrm{C}$. The supernatants were collected to determine protein concentration by the BCA protein assay (Pierce, Rockford, IL, USA) using bovine serum albumin (BSA) as the standard. Then protein samples $(30 \mathrm{mg}$ ) were mixed with an equal amount of $2 \mathrm{x}$ Laemmli buffer and boiled for $5 \mathrm{~min}$, subsequently subjected to SDS-PAGE. The proteins were transferred to nitrocellulose membranes (Osmonics, Minnetonka, MN, USA), which were blocked with TBS containing $0.1 \%$ Tween-20 (TBST) and 5\% non-fat milk for $1 \mathrm{~h}$ at room temperature, followed by incubation with a specific primary antibody $(1: 1,000)$ in at $4^{\circ} \mathrm{C}$ overnight. After three washes with TBST, the blots were incubated with peroxidase-conjugated $\mathrm{IgG}$ for $1 \mathrm{~h}$ at room temperature, visualized using ECL (Amersham Biosciences, Piscataway, NJ, USA) and quantified by Scion Image Software (Scion Corp., Frederick, MD, USA).

\section{Results}

Effect of DIM on cyclin D1 expression in colorectal cancer cells. To investigate the effect of DIM on cyclin expression in colorectal cancer cells, we treated HCT-116 cells with DIM at different dose- and time-dependent manner. As a result, DIM downregulated cyclin D1 and D3 expression in a dose- and time-dependent manner (Fig. 1A and B), but not cyclin E. Since DIM treatment exhibited the strongest decrease in cyclin D1 expression, we further investigated the alteration of cyclin D1 expression in other colorectal cancer cells. Various cancer cells were treated with $25 \mu \mathrm{M}$ DIM for $24 \mathrm{~h}$ and cyclin D1 expression was measured. The result indicated that DIM consistently decreased cyclin D1 in SW480, LoVo and CaCo-2 cells (Fig. 1C), suggesting that cyclin D1 downregulation could 
A

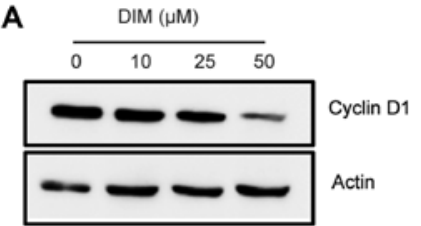

B
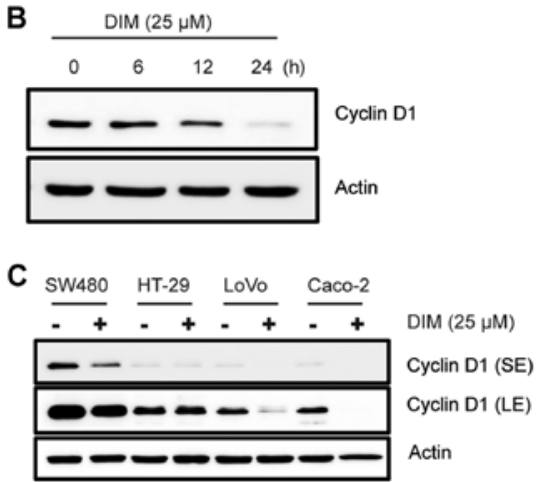

Figure 1. Effect of DIM on cyclin D1 expression in CRC cells. (A and B) HCT116 cells were treated with DIM as indicated dose- and time-dependently. Cells were harvested and the lysates were subjected to western blot analysis using cyclin D1, cyclin D3, cyclin E and actin antibodies. (C) Western blot analysis was carried out to analyze the cell lysates from SW480, HT-29, LoVo and Caco-2 cells treated with DMSO and $25 \mu \mathrm{M}$ DIM for $24 \mathrm{~h}$ using cyclin D1 and actin antibodies. SE, short exposure; LE, long exposure.

A
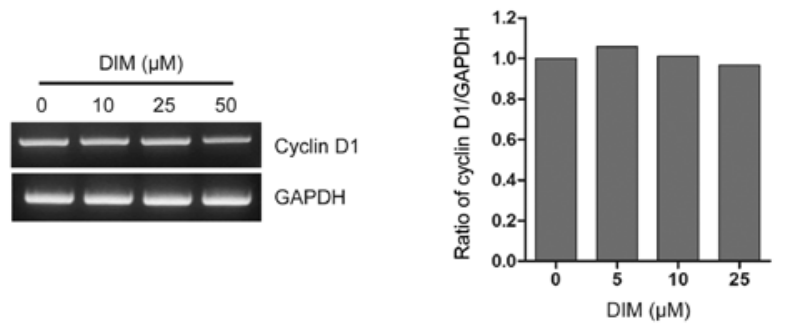

B
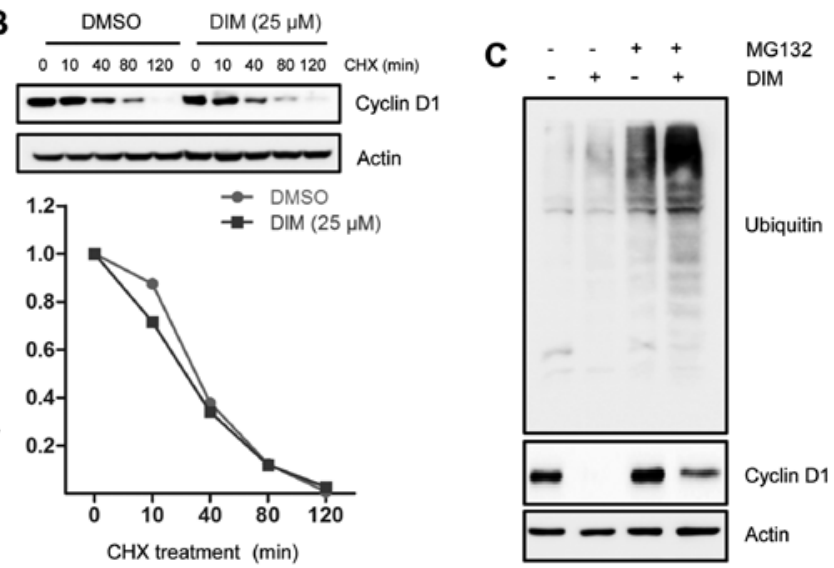

Figure 2. Effect of DIM on cyclin D1 transcriptional and post-transcriptional modulation. (A) HCT-116 cells were treated with $25 \mu \mathrm{M}$ DIM in a timedependent manner. Cells were harvested at 0, 6, 12 and $24 \mathrm{~h}$, and total RNA were isolated and reverse-transcribed into cDNA which was subjected to PCR analysis as described in Materials and methods. Quantitative analysis is shown in the right panel. (B) SW480 cells were pretreated with DMSO and $25 \mu \mathrm{M}$ DIM for $1 \mathrm{~h}$, followed by exposure to $10 \mu \mathrm{g} / \mathrm{ml}$ cycloheximide (CHX). Cells were harvested at the indicated time-point and then subjected to immunoblotting using cyclin D1 antibody (upper panel). Quantitative analysis was carried out by Scion Image Software (lower panel). (C) HCT-116 cells were pretreated with $10 \mu \mathrm{M}$ MG132 for $1 \mathrm{~h}$, followed by treatment with $25 \mu \mathrm{M}$ DIM for $24 \mathrm{~h}$. Western blot analysis was performed with ubiquitin, cyclin D1 and actin antibodies.
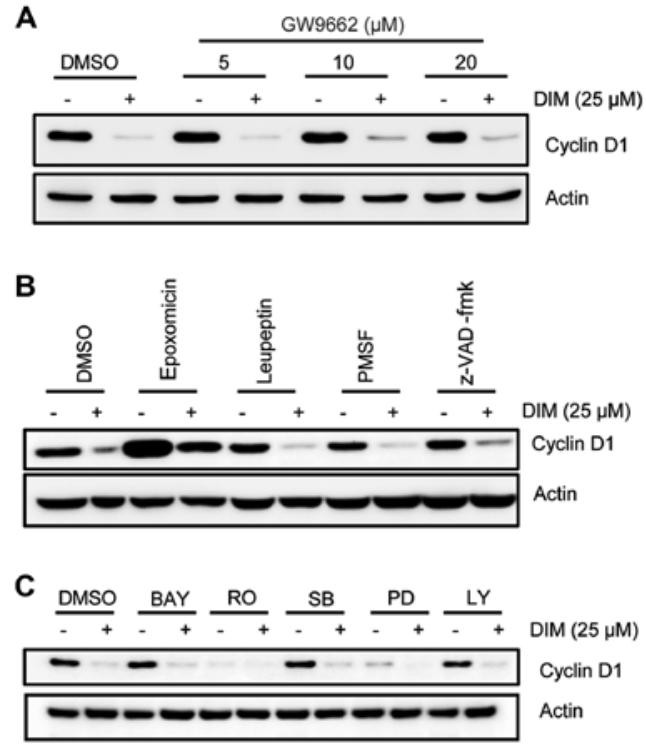

Figure 3. Effect of signal pathways on DIM-mediated cyclin D1 downregulation. (A) HCT-116 cells were pretreated with GW9662 dose-dependently for $1 \mathrm{~h}$, followed by incubation with $25 \mu \mathrm{M}$ DIM for another $24 \mathrm{~h}$. Cell lysates were analyzed using cyclin D1 antibody. Actin was as a loading control. (B and C) HCT-116 cells were pretreated with $1 \mu \mathrm{M}$ epoxomicin, $100 \mu \mathrm{M}$ leupeptin, $1 \mathrm{mM}$ PMSF, $50 \mu \mathrm{M}$ z-VAD-fmk, $10 \mu \mathrm{M}$ BAY11-7082 (BAY), $10 \mu \mathrm{M}$ rottlerin (RO), $10 \mu \mathrm{M}$ SB203580(SB), $40 \mu \mathrm{M}$ PD98059 (PD), $10 \mu \mathrm{M}$ LY294002 (LY) for $1 \mathrm{~h}$, then incubated with $25 \mu \mathrm{M}$ DIM for $24 \mathrm{~h}$. The lysates were analyzed by western blot analysis.

be a potential mechanism for the anti-proliferative effect of DIM.

Effect of DIM on cyclin D1 mRNA expression and protein stability. To better understand the underlying mechanism by which DIM reduced cyclin D1 expression, we assessed the effect of DIM on cyclin D1 mRNA expression and protein stability. As seen in Fig. 2A, the level of cyclin D1 mRNA was not altered in the presence of DIM. Moreover, we examined cyclin D1 protein stability, showing that DIM did not affect its turnover rate when blocking protein synthesis (Fig. 2B). This result was further examined using proteasomal inhibitor MG-132. As shown in Fig. 2C, a proteasome inhibitor MG-132 marginally restored cyclin D1 expression in the presence of DIM. It is suggested that DIM marginally affects proteasomal pathway for cyclin D1 degradation, although the level of ubiquitinated proteins is increased by blocking ubiquitinproteasome pathway.

Taking together, DIM deceased cyclin D1 independent of degradation pathway and these data implicated that DIM-induced cyclin D1 downregulation could occur at the translational level.

Effects of signaling pathways on DIM-mediated cyclin D1 downregulation. DIM has been reported to inhibit proliferation of colorectal cancer cells through activating PPAR $\gamma$ activity (15). Thus, PPAR $\gamma$ antagonist was treated in HCT-116 cells. As shown in Fig. 3A, cyclin D1 was not affected by a PPAR $\gamma$ antagonist GW9662 in the presence of DIM, indicating that its reduction was not associated with PPAR $\gamma$ activation. To further examine other possible signaling pathways mediated 

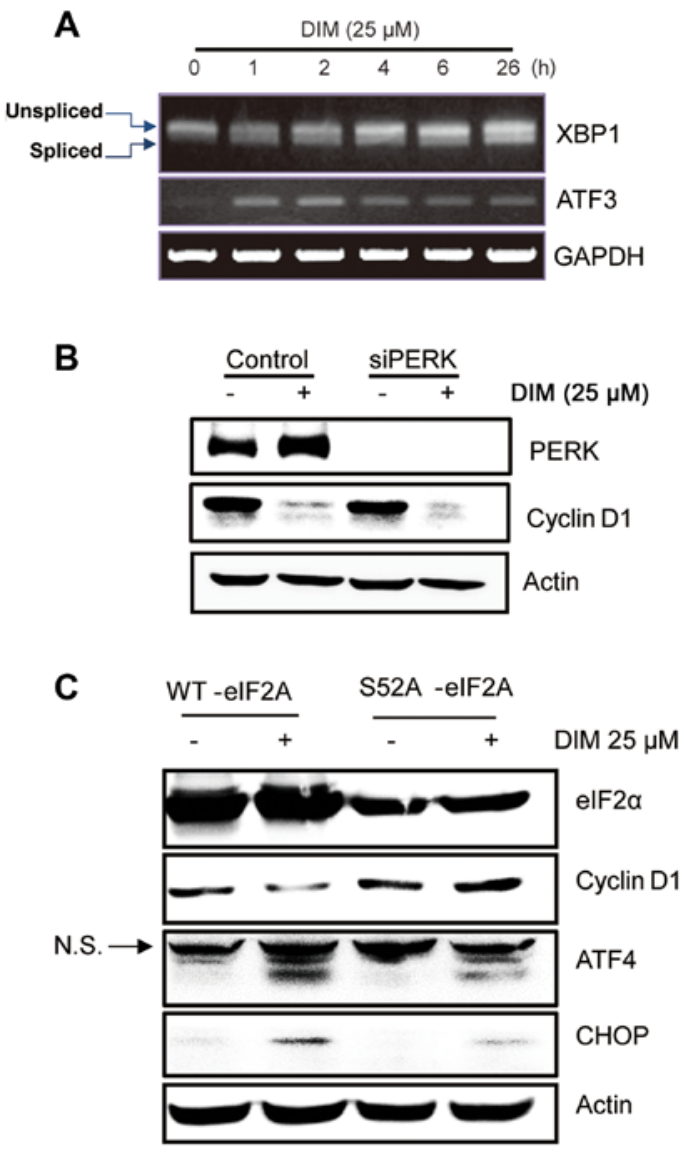

Figure 4. Effect of ER stress response on cyclin D1 downregulation by DIM. (A) HCT-116 cells were treated with $25 \mu$ M DIM time-dependently. Cells were harvested at indicated time. Reverse-transcription PCR was performed for analysis of XBP1 and ATF3 expression as described in Materials and methods. (B) Control and PERK siRNA were transfected into HCT-116 cells, followed by DIM treatment for $24 \mathrm{~h}$. Cells were harvested and the lysates were subjected to western blot analysis using PERK and cyclin D1 antibodies. (C) HCT-116 cells were respectively transfected with wild-type eIF2A and S52A-eIF2A for $24 \mathrm{~h}$, followed by treatment with DMSO and $25 \mu \mathrm{M}$ DIM for $24 \mathrm{~h}$. The lysates were examined by western blot analysis using elF2 $\alpha$, CHOP, cyclin D1, ATF4 and actin antibodies.

by the downregulation of cyclin D1 by DIM, we treated cells with various inhibitors, including proteasome inhibitor epoxomicin, cysteine protease inhibitor leupeptin, serine protease inhibitor PMSF, pan-caspase inhibitor z-VAD-fmk, NF- $\kappa \mathrm{B}$ inhibitor Bay 11-7082 (BAY), PKC inhibitor Rottlerin (RO), p38MAPK inhibitor SB203580 (SB), ERK inhibitor PD98059 (PD), and PI3K inhibitor LY294002 (LY). As shown in Fig. 3B, epoxomicin apparently increased basal cyclin D1 expression, suggesting basal cyclin D1 was mainly regulated by the proteasome pathway. However, compared to treatment with different inhibitors alone, co-treated with DIM consistently downregulated cyclin D1 expression, except of RO and PD compound because they decreased basal cyclin D1 expression as well (Fig. 3B and C). Collectively, PPAR $\gamma$ and several kinase pathways seemed not to affect DIM-mediated cyclin D1 downregulation.

Effect of ER stress response on cyclin D1 downregulation by $D I M$. To further elucidate the signaling pathways involved in DIM-induced cyclin D1 translation suppression, we examined whether DIM triggered ER stress response of colorectal cancer cells. It is known that ER stress inhibits the translation of cyclin D1, mediated by the phosphorylation of eIF $2 \alpha$. As expected, DIM induced x-box-bindingprotein-1 (XBP-1) mRNA splicing and ATF3 expression in a time-dependent manner (Fig. 4A). These are the markers for ER stress. Next, we measured whether PERK, one of upstream component of elF2 $\alpha$, contributes to DIM-mediated cyclin D1 downregulation. Blocking PERK did not seem to affect DIM-mediated cyclin D1 downregulation (Fig. 4B), indicating that DIM may affect elF $2 \alpha$ by other kinases. Given the critical role of eIF $2 \alpha$ phosphorylation in ER stress-mediated protein translation inhibition, we then asked whether eIF2 $\alpha$ protein phosphorylation mediated cyclin D1 downregulation by DIM. As shown in Fig. 4C, transfecting eIF $2 \alpha$ construct harboring an S52A mutation attenuated ER stress response as assessed by CHOP and ATF4 upregulation, indeed restoring cyclin D1 expression in the presence of DIM. It suggested that ER stress-mediated protein synthesis inhibition played key roles in cyclin D1 downregulation by DIM in CRC cells.

\section{Discussion}

There is a large number of evidence showing that the high intake of cruciferous vegetables is inversely associated with the risk of CRC in humans (16). As a major component of cruciferous vegetables, I3C and its condensation product DIM exhibited anti-tumorigenic effect in different types of cancer cells and in animal models (17). Given the important role of I3C/DIM in cancer chemoprevention, the multiple mechanisms responsible for the anti-proliferative effect of DIM have been proposed, including the regulation of cell cycle regulators such as p21, p27, cyclin D1 or E as well as CKDs 2, 4, 6 , which in part was attributed to the effect of DIM on Sp1 transcriptional activity (18). Herein, we further identified that cyclin D1 was downregulated by DIM via ER-stress-mediated protein synthesis inhibition, which provided new mechanism for the potential chemo-preventive or therapeutic function of DIM in CRC.

It has been documented that I3C induced G1 cell cycle arrest in prostate and breast cancer cells, which is accompanied with cyclin D1 downregulation $(19,20)$. Thus, it is not surprising that we found DIM decreased cyclin D1 in CRC cells. However, cyclin D1 in HT-29 cells was not substantially altered in the presence of DIM (Fig. 1C), which was in agreement with a previous report (15) that DIM analogues like DIM-C-pPhCF3 and DIM-C-pPhtBu did not affect the expression of cyclin D1 in HCT-15 or HT-29 cells, suggesting that DIM-induced cyclin D1 downregulation was dependent on cell content.

Several PPAR $\gamma$ agonists have been reported to downregulate cyclin D1 expression at transcriptional or post-transcriptional level as part of the mechanism for causing G1 cell cycle arrest or growth inhibition through receptor-dependent and -independent pathways. We have previously observed that PPAR $\alpha / \gamma$ dual ligand MCC-555 decreased cyclin D1 in pancreatic cancer cells in a PPAR $\gamma$ dependent manner (21). In contrast, our finding in the present study demonstrated that DIM altered cyclin D1 expression 
independent of PPAR $\gamma$ activity. In addition, a DIM derivate did not affect cyclin D1 expression although it activated PPAR $\gamma$ in CRC cells (15). The evidence suggested that DIM could exhibit multiple growth-inhibitory mechanisms which varies in different types of cancer cells, and is dependent on cell content.

The ubiquitin-proteasome degradation pathway plays a key role in modulating cell cycle regulators, including cyclin D1, since they are short-life proteins. Not surprisingly, the 26s proteasome inhibitors MG-132 and epoximicin both increased basal cyclin D1 expression of HCT-116 cells in the present study (Figs. 2C and 3B). A large number of chemicals or drugs have been documented to trigger cyclin D1 degradation through proteasome pathway (13). DIM and its derivate have also been reported to reduce cyclin D1 in MCF-7 and MDA-MB231 cells, which was blocked by proteasome inhibitor MG-132 (14). Our finding showed that proteasome inhibitors failed to completely reverse cyclin D1 downregulation in the presence of DIM, indicating DIM did not induce proteasomedependent cyclin D1 degradation (Fig. 2C). However, we did observe that DIM increased the level of ubiquitinated protein when proteasome activity was blocked, suggesting DIM could target other proteins through activating ubiquitin-proteasome pathway. Indeed, Li et al (22) reported that DIM selectively induced proteasomal degradation of class I histone deacetylases in CRC cells. Moreover, various pathway inhibitors failed to restore cell cyclin D1 expression in the presence of DIM. Although these inhibitors have previously been verified to block specific pathways by our group $(23,24)$, they could harbor other non-identified activities, and induce cell stress by themselves. Therefore, the signaling pathways involved in cyclin D1 downregulation by DIM should be further carefully ruled out.

Accumulating evidence showed that chemicals or drugs harboring anticancer activity were able to trigger ER stress, which contributes to cell cycle arrest and apoptosis. DIM induced apoptosis through ER stress-mediated upregulation of DR5 in pancreatic cancer cells (6). We also reported DIM increased ATF3 and ATF4 expression in CRC cells, both of which can be considered as markers of ER stress. ER stressmediated eIF $2 \alpha$ phosphorylation leads to nearly global protein repression by limiting the delivery of initiator Met-tRNAi to translation machinery, including cyclin D1 (25). In the present study we employed constitutively active eIF $2 \alpha$ construct (S52A mutation) which had been documented to attenuate ER stress response in HCT-116 cells (26). As expected, transfection of mutant eIF2 $\alpha$ construct weakened DIM-induced ER stress as evaluated by examining CHOP and ATF4 expression, and rescued cyclin D1 expression, suggesting DIM halted cyclin D1 protein translation by triggering ER stress. Moreover, the results were also supported by the observation that neither cyclin D1 mRNA expression nor protein stability was affected by DIM. However, the detailed evidence that DIM inhibited cyclin D1 protein synthesis remains to be further investigated.

Taken together, we presented here that DIM modulated cyclin D1 through activating ER stress response, therefore, providing new insight into its anti-proliferative effect on CRC cells. Given the multiple signaling pathways induced by ER stress, it would be meaningful to clarify DIM-induced ER stress pathways and identify potential anticancer molecules in ongoing investigations.

\section{Acknowledgements}

The present study was supported by the Research Resettlement Fund for the new faculty of Seoul National University and partially supported by the Research Institute for Veterinary Science, Seoul National University. The study was also supported in part by the Program in Organizational or Personal Cooperation with Foreign Counterparts (no. 2010630161), the China Scholarship Council, China.

\section{References}

1. Lee KW, Bode AM and Dong Z: Molecular targets of phytochemicals for cancer prevention. Nat Rev Cancer 11: 211-218, 2011.

2. Kassie F, Uhl M, Rabot S, Grasl-Kraupp B, Verkerk R, Kundi M, Chabicovsky M, Schulte-Hermann R and Knasmüller S: Chemoprevention of 2-amino-3-methylimidazo[4,5-f]quinoline (IQ)-induced colonic and hepatic preneoplastic lesions in the F344 rat by cruciferous vegetables administered simultaneously with the carcinogen. Carcinogenesis 24: 255-261, 2003.

3. Weng JR, Tsai CH, Kulp SK and Chen CS: Indole-3-carbinol as a chemopreventive and anti-cancer agent. Cancer Lett 262: 153-163, 2008.

4. Lee SH, Min KW, Zhang X and Baek SJ: 3,3'-diindolylmethane induces activating transcription factor 3 (ATF3) via ATF4 in human colorectal cancer cells. J Nutr Biochem 24: 664-671, 2013.

5. Nachshon-Kedmi M, Yannai S, Haj A and Fares FA: Indole-3carbinol and 3,3 -diindolylmethane induce apoptosis in human prostate cancer cells. Food Chem Toxicol 41: 745-752, 2003.

6. Abdelrahim M, Newman K, Vanderlaag K, Samudio I and Safe S: 3,3'-diindolylmethane (DIM) and its derivatives induce apoptosis in pancreatic cancer cells through endoplasmic reticulum stressdependent upregulation of DR5. Carcinogenesis 27: 717-728, 2006.

7. Rahman KW and Sarkar FH: Inhibition of nuclear translocation of nuclear factor- $\{\mathrm{kappa}\} \mathrm{B}$ contributes to 3,3'-diindolylmethaneinduced apoptosis in breast cancer cells. Cancer Res 65: 364-371, 2005.

8. Kassouf W, Chintharlapalli S, Abdelrahim M, Nelkin G, Safe $S$ and Kamat AM: Inhibition of bladder tumor growth by 1,1-bis(3'-indolyl)-1-(p-substituted phenyl)methanes: A new class of peroxisome proliferator-activated receptor gamma agonists. Cancer Res 66: 412-418, 2006.

9. Gong Y, Firestone GL and Bjeldanes LF: 3,3'-diindolylmethane is a novel topoisomerase IIalpha catalytic inhibitor that induces S-phase retardation and mitotic delay in human hepatoma HepG2 cells. Mol Pharmacol 69: 1320-1327, 2006.

10. Chintharlapalli S, Papineni S, Baek SJ, Liu S and Safe S: 1,1-Bis(3'-indolyl)-1-(p-substituted phenyl)methanes are peroxisome proliferator-activated receptor gamma agonists but decrease HCT-116 colon cancer cell survival through receptor-independent activation of early growth response-1 and nonsteroidal anti-inflammatory drug-activated gene-1. Mol Pharmacol 68: 1782-1792, 2005.

11. Musgrove EA, Caldon CE, Barraclough J, Stone A and Sutherland RL: Cyclin D as a therapeutic target in cancer. Nat Rev Cancer 11: 558-572, 2011.

12. Jirawatnotai S, Hu Y,Michowski W, Elias JE, Becks L, Bienvenu F, Zagozdzon A, Goswami T, Wang YE, Clark AB, et al: A function for cyclin D1 in DNA repair uncovered by protein interactome analyses in human cancers. Nature 474: 230-234, 2011.

13. Alao JP: The regulation of cyclin D1 degradation: Roles in cancer development and the potential for therapeutic invention. Mol Cancer 6: 24, 2007.

14. Vanderlaag K, Samudio I, Burghardt R, Barhoumi R and Safe S: Inhibition of breast cancer cell growth and induction of cell death by 1,1-bis(3'-indolyl)methane (DIM) and 5,5'-dibromoDIM. Cancer Lett 236: 198-212, 2006.

15. Chintharlapalli S, Smith R III, Samudio I, Zhang W and Safe S: 1,1-Bis(3'-indolyl)-1-(p-substituted phenyl)methanes induce peroxisome proliferator-activated receptor gamma-mediated growth inhibition, transactivation, and differentiation markers in colon cancer cells. Cancer Res 64: 5994-6001, 2004. 
16. Wu QJ, Yang Y, Vogtmann E, Wang J, Han LH, Li HL and Xiang YB: Cruciferous vegetables intake and the risk of colorectal cancer: A meta-analysis of observational studies. Ann Oncol 24: 1079-1087, 2013.

17. Banerjee S, Kong D, Wang Z, Bao B, Hillman GG and Sarkar FH: Attenuation of multi-targeted proliferation-linked signaling by 3,3'-diindolylmethane (DIM): From bench to clinic. Mutat Res 728: 47-66, 2011.

18. Firestone GL and Bjeldanes LF: Indole-3-carbinol and 3-3'-diindolylmethane antiproliferative signaling pathways control cell-cycle gene transcription in human breast cancer cells by regulating promoter-Sp1 transcription factor interactions. J Nutr 133 (Suppl): 2448S-2455S, 2003.

19. Hong C, Kim HA, Firestone GL and Bjeldanes LF: 3,3'-Diindolylmethane (DIM) induces a $\mathrm{G}_{1}$ cell cycle arrest in human breast cancer cells that is accompanied by Sp1-mediated activation of $\mathrm{p} 21^{\mathrm{WAF} 1 / \mathrm{CIP} 1}$ expression. Carcinogenesis 23: 1297-1305, 2002.

20. Vivar OI, Lin CL, Firestone GL and Bjeldanes LF: 3,3'-Diindolylmethane induces a $\mathrm{G}_{1}$ arrest in human prostate cancer cells irrespective of androgen receptor and p53 status. Biochem Pharmacol 78: 469-476, 2009.
21. Min KW, Zhang X, Imchen T and Baek SJ: A peroxisome proliferator-activated receptor ligand MCC-555 imparts anti-proliferative response in pancreatic cancer cells by PPARgamma-independent up-regulation of KLF4. Toxicol Appl Pharmacol 263: 225-232, 2012.

22. Li Y, Li X and Guo B: Chemopreventive agent 3,3'-diindolylmethane selectively induces proteasomal degradation of class I histone deacetylases. Cancer Res 70: 646-654, 2010

23. Lee SH, Bahn JH, Whitlock NC and Baek SJ: Activating transcription factor 2 (ATF2) controls tolfenamic acid-induced ATF3 expression via MAP kinase pathways. Oncogene 29: 5182-5192, 2010.

24. Zhang X, Min KW, Wimalasena J and Baek SJ: Cyclin D1 degradation and $\mathrm{p} 21$ induction contribute to growth inhibition of colorectal cancer cells induced by epigallocatechin-3-gallate. J Cancer Res Clin Oncol 138: 2051-2060, 2012.

25. Sonenberg N and Hinnebusch AG: Regulation of translation initiation in eukaryotes: Mechanisms and biological targets. Cell 136: 731-745, 2009

26. Yoon CH, Lee ES, Lim DS and Bae YS: PKR, a p53 target gene, plays a crucial role in the tumor-suppressor function of p53. Proc Natl Acad Sci USA 106: 7852-7857, 2009. 